\title{
Reactive Sulfur Species: Aqueous Chemistry of Sulfenyl Thiocyanates
}

\author{
Michael T. Ashby* and Halikhedkar Aneetha \\ Department of Chemistry and Biochemistry, University of Oklahoma, \\ 620 Parrington Oval, Norman, OK 73019
}

Submitted March 10, 2004 -- Revised Manuscript submitted June 1, 2004

\section{SUPPORTING INFORMATION}

Experimental

Table S1. ${ }^{1} \mathrm{H}$ NMR Chemical Shifts (ppm) of $\gamma$-L-Glutamyl-L-cysteinylglycine (GSH), $\gamma$-L-Glutamyl-L-S-carbamylcysteinylglycine (GSC), and $\gamma$-L-Glutamyl-L-S-thiocyanato-cysteinylglycine (GSSCN) in $\mathrm{D}_{2} \mathrm{O}$

Table S2. ${ }^{1} \mathrm{H}$ NMR Coupling Constants (Hz) of $\gamma$-L-Glutamyl-L-cysteinylglycine (GSH), $\gamma$-L-Glutamyl-L-S-carbamylcysteinylglycine (GSC), and $\gamma$-L-Glutamyl-L-S-thiocyanato-cysteinylglycine (GSSCN) in $\mathrm{D}_{2} \mathrm{O} O$

Table S3. ${ }^{13} \mathrm{C}$ NMR Chemical Shifts (ppm) of $\gamma$-L-Glutamyl-L-cysteinylglycine (GSH), $\gamma$-L-Glutamyl-L-S-carbamylcysteinylglycine (GSC), and $\gamma$-L-Glutamyl-L-S-thiocyanato-cysteinylglycine (GSSCN) in $\mathrm{D}_{2} \mathrm{O}$

Table S4. Crystal data and structure refinement for NTS.

Table S5. Atomic coordinates ( $x 10^{4}$ ) and equivalent isotropic displacement parameters $\left(\AA^{2} \times 10^{3}\right)$ for NTS.

Table S6. Bond lengths $[\AA]$ and angles $\left[^{\circ}\right]$ for NTS.

Table S7. Anisotropic displacement parameters $\left(\AA^{2} \times 10^{3}\right)$ for NTS.

Table S8. Hydrogen coordinates ( x 104) and isotropic displacement parameters $\left(\AA^{2} \times 10^{3}\right)$ for NTS.

Table S9. Torsion angles $\left[^{\circ}\right]$ for NTS.

Figure S1. Thermal ellipoid drawing of the crystal structure of NTS at the $50 \%$ level with the labeling scheme.

Figure S2. Crystal packing diagram of the crystal structure of NTS as viewed along the $\mathbf{b}$ axis. 


\section{EXPERIMENTAL}

All reagents were of the highest purity available and used as received from SigmaAldrich unless stated otherwise. Infrared measurements were made using a Bruker IFS 66/S FTIR fitted with a Whatman FT-IR Purge Gas Generator (model 75-52). The cell was a commercial flow-through design with $\mathrm{CaF}_{2}$ windows and a $0.015 \mathrm{~mm}$ Teflon spacer. UV-vis spectra were collected using a HP8452A diode array instrument. $\mathrm{pH}$ measurements were made with an Orion ionAnalyzer EA920 using a $\mathrm{Ag} / \mathrm{AgCl}$ combination $\mathrm{pH}$ electrode. $\mathrm{pD}$ measurements in $\mathrm{D}_{2} \mathrm{O}$ were made using the same $\mathrm{pH}$ electrode by adding 0.4 units to the measurement. NMR spectra were collected with a Varian XL-300 spectrometer using DSS $(\delta=0.015 \mathrm{ppm})$ to reference the ${ }^{1} \mathrm{H}$ spectra and dioxane $(\delta=67.4 \mathrm{ppm})$ for the ${ }^{13} \mathrm{C}$ spectra. ES-MS spectra were collected with a Micromass Qtof in positive ion mode at $\mathrm{pH}=2.5$. X-ray data were measured with a Bruker Apex diffractometer using the conditions that are listed in Table S4.

In situ synthesis of sulfenyl thiocyanates. The concentration of $\mathrm{Br}_{2}$ in water-saturated $\mathrm{CCl}_{4}$ was determined spectrophotometrically $\left(\varepsilon_{400 \mathrm{~nm}}=160 \mathrm{M}^{-1} \mathrm{~cm}^{-1}\right)$. Excess $\mathrm{Pb}(\mathrm{SCN})_{2}$ was added and the resulting slurry was vortexed until the solution became colorless. The $\mathrm{Pb}$ salts were removed by centrifuging and decanting. The concentration of $(\mathrm{SCN})_{2}$ in $\mathrm{CCl}_{4}$ was confirmed spectrophotometrically $\left(\varepsilon_{296 \mathrm{~nm}}=140 \mathrm{M}^{-1} \mathrm{~cm}^{-1}\right)$. A solution of thiol (typically $50 \mathrm{mM}$ ) in $1 \mathrm{M} \mathrm{DCl}$ was treated with an aliquot of the $\mathrm{CCl}_{4}$ solution. The twophase solution was vortexed $2 \mathrm{~min}$, centrifuged, and the top layer was transferred to a NMR tube.

L-S-thiocyanato-penicillamine (PENSCN): $\quad{ }^{1} \mathrm{H}$ NMR (1M DCl, $\left.300 \mathrm{MHz}\right) \delta 4.35$ (s, $1 \mathrm{H}_{\text {PEN } \alpha}$ ), 1.75 (s, $\left.3 \mathrm{H}_{\text {PEN Me }}\right) 1.64$ (s, $\left.3 \mathrm{H}_{\text {PEN Me }}\right) ;{ }^{13} \mathrm{C}\{1 \mathrm{H}\}$ NMR (1M DCl, $\left.75.5 \mathrm{MHz}\right) \delta$ 169.3, 114.7 (SCN), 59.8, 53.1, 26.2, 23.1; MS (ES-MS+) calcd for $\mathrm{C}_{6} \mathrm{H}_{10} \mathrm{~N}_{2} \mathrm{O}_{2} \mathrm{~S}_{2} \mathrm{~m} / \mathrm{z}$ 206, found $\mathrm{m} / \mathrm{z} 207$ for $\mathrm{M}+\mathrm{H}^{+}$. IR ( $1 \mathrm{M} \mathrm{HCl}, 15 \mu \mathrm{m}$ pathlength) $2161 \mathrm{~cm}^{-1}\left(v_{\mathrm{CN}}\right)$. UV-vis $(1 \mathrm{M} \mathrm{HCl}) \varepsilon_{280 \mathrm{~nm}}=188(14) \mathrm{M}^{-1} \mathrm{~cm}^{-1}$.

$\gamma$-L-Glutamyl-L-S-thiocyanato-cysteinylglycine (GSSCN): $\quad{ }^{1} \mathrm{H}$ NMR (1M DCl, 300 $\mathrm{MHz}) \delta 4.86\left(\mathrm{dd},{ }^{3} \mathrm{~J}_{\text {Cys } \alpha-\mathrm{H}, \text { Cys } \beta-\mathrm{Ha}}=4.5,{ }^{3} \mathrm{~J}_{\text {Cys } \alpha-\mathrm{H}, \text { Cys } \beta-\mathrm{Hb}}=9.2 \mathrm{~Hz}, 1 \mathrm{H}_{\mathrm{Cys}} \alpha\right), 4.18\left(\mathrm{dd},{ }^{3} \mathrm{~J}_{\mathrm{Glu}}\right.$ $\alpha$-H,Glu $\beta$-Ha $\left.=6.0,{ }^{3} \mathrm{~J}_{\text {Glu } \alpha-\mathrm{H}, \mathrm{Glu} \beta-\mathrm{Hb}}=6.0 \mathrm{~Hz}, 1 \mathrm{H}_{\mathrm{Glu} \alpha}\right), 4.06\left(\mathrm{~s}, 2 \mathrm{H}_{\mathrm{Gly} \alpha}\right), 3.64\left(\mathrm{dd},{ }^{2} \mathrm{~J}_{\mathrm{Cys} \beta} \beta\right.$-Ha, Cys $\beta$-Hb $\left.=15.0,{ }^{3} \mathrm{~J}_{\text {Cys } \alpha-\mathrm{H} \text {, Суs } \beta-\mathrm{Ha}}=4.5 \mathrm{~Hz}, 1 \mathrm{H}_{\text {Cys } \beta-\mathrm{Hb}}\right), 3.39\left(\mathrm{dd},{ }^{2} \mathrm{~J}_{\text {Сys } \beta-\mathrm{Ha}, \text { Сys } \beta-\mathrm{Hb}}=15.0,{ }^{3} \mathrm{~J}\right.$ Cys $\alpha-\mathrm{H}$, Сys $\beta$-Ha $\left.=9.2 \mathrm{~Hz}, 1 \mathrm{H}_{\text {Cys } \beta-\mathrm{Ha}}\right), 2.69\left(\mathrm{ddd},{ }^{3} \mathrm{~J}_{\mathrm{Glu} \beta-\mathrm{Ha}, \mathrm{Glu} \gamma-\mathrm{Hb}}=6.0,{ }^{3} \mathrm{~J}_{\mathrm{Glu} \beta-\mathrm{Hb}, \mathrm{Glu} \gamma-\mathrm{Hb}}=6.0\right.$ $\left.\mathrm{Hz}, 1 \mathrm{H}_{\mathrm{Glu} \gamma-\mathrm{Hb}}\right), 2.65\left(\mathrm{ddd},{ }^{3} \mathrm{~J}_{\mathrm{Glu} \beta-\mathrm{Ha}, \mathrm{Glu} \gamma-\mathrm{Ha}}=6.0,{ }^{3} \mathrm{~J}_{\mathrm{Glu} \beta-\mathrm{Hb}, \mathrm{Glu} \gamma-\mathrm{Ha}}=6.0 \mathrm{~Hz}, 1 \mathrm{H}_{\mathrm{Glu} \gamma-\mathrm{Ha}}\right), 2.29$

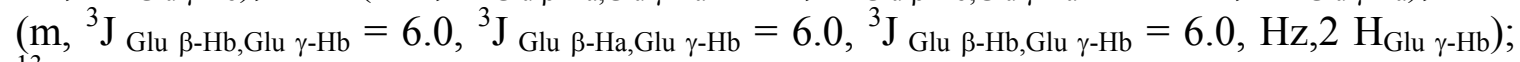
${ }^{13} \mathrm{C}\{1 \mathrm{H}\}$ NMR $(1 \mathrm{M} \mathrm{DCl}, 75.5 \mathrm{MHz}) \delta 175.1,173.4,172.5,171.8,114.9(\mathrm{SCN}), 53.3$, 52.9, 42.1, 40.6, 31.9, 26.1; MS (ES-MS+) calcd for $\mathrm{C}_{11} \mathrm{H}_{16} \mathrm{~N}_{4} \mathrm{O}_{6} \mathrm{~S}_{2} \mathrm{~m} / \mathrm{z} 364$, found $\mathrm{m} / \mathrm{z}$ 365 for $\mathrm{M}+\mathrm{H}^{+}$. IR (1M HCl, $15 \mu \mathrm{m}$ pathlength) $2147 \mathrm{~cm}^{-1}\left(v_{\mathrm{CN}}\right)$. UV-vis $(1 \mathrm{M} \mathrm{HCl}) \varepsilon_{280 \mathrm{~nm}}$ $=274(11) \mathrm{M}^{-1} \mathrm{~cm}^{-1}$.

In situ synthesis of S-carbamyl derivative of GSH. Cyanate is produced as a decomposition product of hypothiocyanite. Since $\mathrm{OCN}^{-}$is known to react with thiols, we synthesized the GSH derivative (GSC) to compare its chemical and spectral features with GSSCN. A solution of GSH (typically $50 \mathrm{mM}$ ) in $\mathrm{D}_{2} \mathrm{O}$ at $\mathrm{pD}=7$ was treated with 1.1 equiv. of $\mathrm{NaOCN}$. The reaction to produce $\gamma$-L-glutamyl-L-S-carbamylcysteinylglycine (GSC) was complete within minutes. The $\mathrm{pD}$ was adjusted to 7 with $\mathrm{DCl}$. The reaction 
is reversible and GSH can be recovered by acidifying the solution, thereby destroying the $\mathrm{OCN}^{-}$. GSC does not exist under the same (acidic) conditions as GSSCN.

$\gamma$-L-Glutamyl-L-S-carbamylcysteinylglycine (GSC): $\quad{ }^{1} \mathrm{H}$ NMR $(\mathrm{pD}=7,300 \mathrm{MHz}) \delta$ $4.66\left(\mathrm{dd},{ }^{3} \mathrm{~J}_{\text {Cys } \alpha-\mathrm{H} \text {, Сys } \beta-\mathrm{Ha}}=4.4,{ }^{3} \mathrm{~J}_{\text {Cys } \alpha-\mathrm{H}, \text { Cys } \beta-\mathrm{Hb}}=7.8 \mathrm{~Hz}, 1 \mathrm{H}_{\text {Cys } \alpha}\right), 3.76\left(\mathrm{dd},{ }^{3} \mathrm{~J}_{\text {Glu } \alpha-\mathrm{H}, \mathrm{Glu} \beta-}\right.$ $\left.\mathrm{Ha}=6.9,{ }^{3} \mathrm{~J}_{\mathrm{Glu} \alpha-\mathrm{H}, \mathrm{Glu} \beta-\mathrm{Hb}}=6.9 \mathrm{~Hz}, 1 \mathrm{H}_{\mathrm{Glu} \alpha}\right), 3.78\left(\mathrm{~s}, 2 \mathrm{H}_{\mathrm{Gly} \alpha}\right), 3.43\left(\mathrm{dd},{ }^{2} \mathrm{~J}_{\text {Cys } \beta-\mathrm{Ha}, \text { Сys } \beta-\mathrm{Hb}}=\right.$ $\left.14.5,{ }^{3} \mathrm{~J}_{\text {Cys } \alpha-\mathrm{H}, \text { Суs } \beta-\mathrm{Ha}}=4.4 \mathrm{~Hz}, 1 \mathrm{H}_{\text {Сys } \beta-\mathrm{Hb}}\right), 3.19\left(\mathrm{dd},{ }^{2} \mathrm{~J}_{\text {Суs } \beta-\mathrm{Ha}, \text { Суs } \beta-\mathrm{Hb}}=14.5,{ }^{3} \mathrm{~J}_{\text {Суs } \alpha-\mathrm{H}, \text { Суs }}\right.$ $\left.\beta-\mathrm{Ha}=7.8, \mathrm{~Hz}, 1 \mathrm{H}_{\mathrm{Cys} \beta-\mathrm{Ha}}\right), 2.52\left(2^{\text {nd }}\right.$ order, ${ }^{3} \mathrm{~J}_{\mathrm{Glu} \beta-\mathrm{Ha}, \mathrm{Glu} \gamma-\mathrm{Hb}}=7.7,{ }^{3} \mathrm{~J}$ Glu $\beta$-Hb,Glu $\gamma-\mathrm{Hb}=7.7$

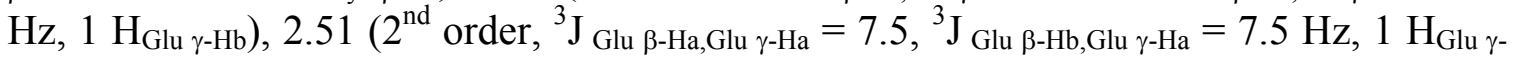
$\mathrm{Ha}), 2.16\left(\mathrm{~m},{ }^{3} \mathrm{~J}_{\mathrm{Glu} \beta} \beta-\mathrm{Hb}, \mathrm{Glu} \gamma-\mathrm{Hb}=7.7,{ }^{3} \mathrm{~J}_{\mathrm{Glu} \beta-\mathrm{Ha}, \mathrm{Glu} \gamma-\mathrm{Hb}}=7.5, \mathrm{~Hz}, 2 \mathrm{H}_{\mathrm{Glu} \gamma-\mathrm{Hb}}\right) ;{ }^{13} \mathrm{C}\{1 \mathrm{H}\} \mathrm{NMR}$ $(\mathrm{pD}=7,75.5 \mathrm{MHz}) \delta 175.7,174.9,177.3,177.2,171.8(\mathrm{SCON}), 55.0,54.5,44.2,32.3$, 31.6, 27.0.

Synthesis of NTS. NBS ( $2 \mathrm{mmol})$ and $\mathrm{NaSCN}(3 \mathrm{mmol})$ were stirred in dry $\mathrm{CH}_{2} \mathrm{Cl}_{2}(15$ $\mathrm{mL})$ for $2 \mathrm{~h}$. The suspension was filtered to remove $\mathrm{NaBr}$. Evaporation of the solvent yielded NTS as a pale yellow solid. Recrystallized NTS $\left(\mathrm{CH}_{2} \mathrm{Cl}_{2} /\right.$ pentane $)$ is colorless. The crude NTS, contaminated by traces of succinimide that were barely detectable by ${ }^{1} \mathrm{H}$ NMR, was used without further purification. ${ }^{1} \mathrm{H}$ NMR $\left(\mathrm{CD}_{2} \mathrm{Cl}_{2}, 300 \mathrm{MHz}\right) \delta 2.95(\mathrm{~s}, 4$ $\mathrm{H}) ;{ }^{13} \mathrm{C}\{1 \mathrm{H}\}$ NMR $\left(\mathrm{CD}_{2} \mathrm{Cl}_{2}, 75.5 \mathrm{MHz}\right) \delta 173.7,108.8,29.2 ; \mathrm{IR}\left(\mathrm{CH}_{2} \mathrm{Cl}_{2}\right) 2160 \mathrm{~cm}^{-1}$ $\left(v_{\mathrm{CN}}\right)$; IR (Nujol) $2173 \mathrm{~cm}^{-1}\left(v_{\mathrm{CN}}\right)$.

X-ray Crystal Structure of NTS. Crystals of NTS were grown by solvent diffusion of pentane into a solution of NTS in $\mathrm{CH}_{2} \mathrm{Cl}_{2}$. The data were collected at 295(2) $\mathrm{K}$ on a Bruker Apex diffractometer [1] using $\operatorname{MoK} \alpha .(\lambda=0.71073 \mathrm{~A})$ radiation. Intensity data, which approximately covered the full sphere of the reciprocal space, were measured as a series of $\omega$ oscillation frames each $0.3^{\circ}$ for $17 \mathrm{sec} /$ frame. The detector was operated in 512 x 512 mode and was positioned $6.12 \mathrm{~cm}$ from the crystal. Coverage of unique data was $99.8 \%$ complete to $54.2^{\circ}(2 \theta)$. Cell parameters were determined from a non-linear least squares fit of 7983 reflections in the range of $2.6<\theta<25.9^{\circ}$. A total of 13779 reflections were measured. The data were corrected for absorption by multi-scan method form equivalent reflections [1] giving minimum and maximum transmission of 0.8557 and 0.9669 . The data were merged to form a set of 1450 unique reflections with $\mathrm{R}(\mathrm{int})=$ 0.018 .

The structure was solved by the direct method using SHELXTL system [2], and refined by full-matrix least squares on $\mathrm{F}^{2}$ using all reflections. All the non-hydrogen atoms were refined anisotropically. All the hydrogen atoms were included with idealized parameters.

Final R $1=0.039$ is based on 1345 "observed reflections" $[\mathrm{I}>2 \sigma(\mathrm{I})]$, and $\mathrm{wR}^{2}=0.113$ is based on all reflections (1450 unique data). Details of the crystal data are given in Table S4. Thermal ellipsoids are drawn at 40\% level for figures S1 and S2. Other relevant data are summarized in Tables S5-S9.

\section{REFERENCES}

1. Bruker (2002) SMART (version 5.625), SAINT-plus (version 6.28A) and SADABS (version 2.03), Bruker AXS Inc., Madison, Wisconsin.

2. Bruker (1997) SHELXTL: Version 6.12, Bruker AXS, and Madison, Wisconsin. 
Table S1. $\quad{ }^{1} \mathrm{H}$ NMR Chemical Shifts (ppm) of $\gamma$-L-Glutamyl-Lcysteinylglycine (GSH), $\gamma$-L-Glutamyl-L-S-carbamylcysteinylglycine (GSC), and $\gamma$-L-Glutamyl-L-S-thiocyanatocysteinylglycine (GSSCN) in $\mathrm{D}_{2} \mathrm{O}$.

$\begin{array}{lccc} & \mathrm{GSH}^{\mathrm{a}} & \mathrm{GSC}^{\mathrm{b}} & \mathrm{GSSCN}^{\mathrm{c}} \\ \text { Gly } \alpha-\mathrm{H}_{\mathrm{a}} & 3.767 & 3.778 & 4.06 \\ \text { Gly } \alpha-\mathrm{H}_{\mathrm{b}} & 3.767 & 3.778 & 4.06 \\ \text { Cys } \alpha-\mathrm{H} & 4.560 & 4.664 & 4.86 \\ \text { Cys } \beta-\mathrm{H}_{\mathrm{a}} & 2.922 & 3.189 & 3.39 \\ \text { Cys } \beta-\mathrm{H}_{\mathrm{b}} & 2.959 & 3.428 & 3.64 \\ \text { Glu } \alpha-\mathrm{H} & 3.772 & 3.763 & 4.18 \\ \text { Glu } \beta-\mathrm{H}_{\mathrm{a}} & 2.155 & 2.162 & 2.29 \\ \text { Glu } \beta-\mathrm{H}_{\mathrm{b}} & 2.155 & 2.162 & 2.29 \\ \text { Glu } \gamma-\mathrm{H}_{\mathrm{a}} & 2.532 & 2.510 & 2.65 \\ \text { Glu } \gamma-\mathrm{H}_{\mathrm{b}} & 2.561 & 2.520 & 2.69 \\ \text { a } 50 \mathrm{mM} \mathrm{GSH} \mathrm{in} 100 \mathrm{mM} \mathrm{pH}=7 \text { buffer. }{ }^{\mathrm{b}} \text { Equilibrium solution of 50 } \\ \text { mM GSH and 50 mM NaNCO in } 100 \mathrm{mM} \mathrm{pH} \mathrm{=7} \mathrm{buffer.}{ }^{\mathrm{c}} 50 \mathrm{mM} \\ \text { GSSCN in } 1 \mathrm{~m} \mathrm{DCl.}\end{array}$


Table S2. ${ }^{1} \mathrm{H}$ NMR Coupling Constants (Hz) of $\gamma$-L-Glutamyl-Lcysteinylglycine (GSH), $\gamma$-L-Glutamyl-L-S-carbamylcysteinylglycine (GSC), and $\gamma$-L-Glutamyl-L-S-thiocyanato-cysteinylglycine (GSSCN) in $\mathrm{D}_{2} \mathrm{O}$.

$\begin{array}{llll} & \mathrm{GSH}^{\mathrm{a}} & \mathrm{GSC}^{\mathrm{b}} & \mathrm{GSSCN}^{\mathrm{c}} \\ { }^{3} \mathrm{~J}_{\mathrm{Cys} \alpha-\mathrm{H}, \text { Cys } \beta-\mathrm{Ha}} & 7.3 & 7.8 & 9.2 \\ { }^{3} \mathrm{~J}_{\mathrm{Cys} \alpha-\mathrm{H}, \mathrm{Cys} \beta-\mathrm{Hb}} & 4.9 & 4.4 & 4.5 \\ { }^{2} \mathrm{~J}_{\mathrm{Cys} \beta-\mathrm{Ha}, \mathrm{Cys} \beta-\mathrm{Hb}} & - & 14.5 & 15.0 \\ { }^{3} \mathrm{~J}_{\mathrm{Glu} \alpha-\mathrm{H}, \mathrm{Glu} \beta-\mathrm{Ha}} & 6.3 & 6.9 & 6.0 \\ { }^{3} \mathrm{~J}_{\mathrm{Glu} \alpha-\mathrm{H}, \mathrm{Glu} \beta-\mathrm{Hb}} & 6.3 & 6.9 & 6.0 \\ { }^{2} \mathrm{~J}_{\mathrm{Glu} \beta-\mathrm{Ha}, \mathrm{Glu} \beta-\mathrm{Hb}} & - & - & - \\ { }^{3} \mathrm{~J}_{\mathrm{Glu} \beta-\mathrm{Ha}, \mathrm{Glu} \gamma-\mathrm{Ha}} & 6.3 & 7.5 & 6.0 \\ { }^{3} \mathrm{~J}_{\mathrm{Glu} \beta-\mathrm{Ha}, \mathrm{Glu} \gamma-\mathrm{Hb}} & 6.3 & 7.7 & 6.0 \\ { }^{3} \mathrm{~J}_{\mathrm{Glu} \beta-\mathrm{Hb}, \mathrm{Glu} \gamma-\mathrm{Ha}} & 6.3 & 7.5 & 6.0 \\ { }^{3} \mathrm{~J}_{\mathrm{Glu} \beta-\mathrm{Hb}, \mathrm{Glu} \gamma-\mathrm{Hb}} & 6.3 & 7.7 & 6.0 \\ { }^{3} \mathrm{~J}_{\mathrm{Glu} \gamma-\mathrm{Ha}, \mathrm{Glu} \gamma-\mathrm{Hb}} & - & - & -\end{array}$

a $50 \mathrm{mM} \mathrm{GSH}$ in $100 \mathrm{mM} \mathrm{pH}=7$ buffer. ${ }^{\mathrm{b}}$ Equilibrium solution of 50 $\mathrm{mM}$ GSH and $50 \mathrm{mM} \mathrm{NaNCO}$ in $100 \mathrm{mM} \mathrm{pH}=7$ buffer. ${ }^{\mathrm{c}} 50 \mathrm{mM}$ GSSCN in $1 \mathrm{M} \mathrm{DCl}$. 
Table S3. ${ }^{13} \mathrm{C}$ NMR Chemical Shifts (ppm) of $\gamma$-L-Glutamyl-Lcysteinylglycine (GSH), $\gamma$-L-Glutamyl-L-S-carbamylcysteinylglycine (GSC), and $\gamma$-L-Glutamyl-L-S-thiocyanato-cysteinylglycine (GSSCN) in $\mathrm{D}_{2} \mathrm{O}$.

$\begin{array}{llll} & \text { GSH }^{\mathrm{a}} & \mathrm{GSC}^{\mathrm{b}} & \mathrm{GSSCN}^{\mathrm{c}} \\ \text { Gly COO } & 177.2 & 177.2 & 175.1 \\ \text { Gly } \alpha-\mathrm{C} & 44.5 & 44.2 & 42.1 \\ \text { Cys CON } & 172.8 & 172.3 & 171.8 \\ \text { Cys } \alpha-\mathrm{C} & 56.55 & 54.5 & 53.3 \\ \text { Cys } \beta-\mathrm{C} & 26.51 & 31.6 & 40.6 \\ \text { Glu CON } & 176.0 & 174.9 & 172.5 \\ \text { Glu } \alpha-\mathrm{C} & 55.2 & 55.0 & 52.9 \\ \text { Glu } \beta-\mathrm{C} & 27.2 & 27.0 & 26.1 \\ \text { Glu } \gamma-\mathrm{C} & 32.4 & 32.3 & 31.9 \\ \text { Glu COO } & 175.1 & 175.7 & 173.4 \\ \text { SCON } & - & 171.8 & - \\ \text { SSCN } & - & - & 114.9\end{array}$

${ }^{\text {a }} 50 \mathrm{mM} \mathrm{GSH}$ in $100 \mathrm{mM} \mathrm{pH}=7$ buffer. ${ }^{\mathrm{b}}$ Equilibrium solution of 50 $\mathrm{mM}$ GSH and $50 \mathrm{mM} \mathrm{NaNCO}$ in $100 \mathrm{mM} \mathrm{pH}=7$ buffer. ${ }^{\mathrm{c}} 50 \mathrm{mM}$ GSSCN in $1 \mathrm{M}$ DCl. 
Table S4. Crystal data and structure refinement for NTS.

Empirical formula

Formula weight

Temperature

Wavelength

Crystal system

Space group

Unit cell dimensions

Volume

Z

Density (calculated)

Absorption coefficient

$\mathrm{F}(000)$

Crystal size

Theta range for data collection

Index ranges

Reflections collected

Independent reflections

Completeness to theta $=27.10^{\circ}$

Absorption correction

Max. and min. transmission

Refinement method

Data / restraints / parameters

Goodness-of-fit on $\mathrm{F}^{2}$

Final R indices [I $>2 \operatorname{sigma}(\mathrm{I})]$

$\mathrm{R}$ indices (all data)

Largest diff. peak and hole
$\mathrm{C} 5 \mathrm{H} 4 \mathrm{~N} 2 \mathrm{O} 2 \mathrm{~S}$

156.16

295(2) K

$0.71073 \AA$

Orthorhombic

Pbca

$\begin{array}{ll}a=11.8634(9) \AA & \alpha=90^{\circ} . \\ b=6.4554(5) \AA & \beta=90^{\circ} . \\ c=17.1787(13) \AA & \gamma=90^{\circ} .\end{array}$

1315.60(17) $\AA^{3}$

8

$1.577 \mathrm{Mg} / \mathrm{m}^{3}$

$0.423 \mathrm{~mm}^{-1}$

640

$0.38 \times 0.18 \times 0.08 \mathrm{~mm}^{3}$

2.93 to $27.10^{\circ}$.

$-15<=\mathrm{h}<=15,-8<=\mathrm{k}<=8,-22<=\mathrm{l}<=22$

13779

$1450[\mathrm{R}(\mathrm{int})=0.0175]$

$99.8 \%$

Semi-empirical from equivalents

0.9669 and 0.8557

Full-matrix least-squares on $\mathrm{F}^{2}$

$1450 / 0 / 91$

1.051

$\mathrm{R} 1=0.0389, \mathrm{wR} 2=0.1106$

$\mathrm{R} 1=0.0411, \mathrm{wR} 2=0.1130$

0.688 and -0.493 e. $\AA^{-3}$ 
Table S5. Atomic coordinates ( $\times 10^{4}$ ) and equivalent isotropic displacement parameters $\left(\AA^{2} \times 10^{3}\right)$ for NTS. U(eq) is defined as one third of the trace of the orthogonalized $\mathrm{U}^{\mathrm{ij}}$ tensor.

\begin{tabular}{lrrrr}
\hline & $\mathrm{x}$ & $\mathrm{y}$ & $\mathrm{z}$ & $\mathrm{U}(\mathrm{eq})$ \\
\hline $\mathrm{S}(1)$ & $8350(1)$ & $1237(1)$ & $3694(1)$ & $49(1)$ \\
$\mathrm{O}(1)$ & $10565(1)$ & $25(2)$ & $2899(1)$ & $59(1)$ \\
$\mathrm{O}(2)$ & $9042(1)$ & $5428(3)$ & $4232(1)$ & $66(1)$ \\
$\mathrm{N}(1)$ & $9603(1)$ & $2453(2)$ & $3617(1)$ & $37(1)$ \\
$\mathrm{N}(2)$ & $8509(2)$ & $-127(3)$ & $5253(1)$ & $64(1)$ \\
$\mathrm{C}(1)$ & $10545(2)$ & $1672(3)$ & $3218(1)$ & $40(1)$ \\
$\mathrm{C}(2)$ & $11448(2)$ & $3289(3)$ & $3266(1)$ & $47(1)$ \\
$\mathrm{C}(3)$ & $10947(2)$ & $5087(3)$ & $3716(1)$ & $48(1)$ \\
$\mathrm{C}(4)$ & $9759(1)$ & $4466(3)$ & $3900(1)$ & $41(1)$ \\
$\mathrm{C}(5)$ & $8461(2)$ & $395(3)$ & $4626(1)$ & $43(1)$ \\
& & & & \\
\hline
\end{tabular}


Table S6. Bond lengths $[\AA]$ and angles $\left[{ }^{\circ}\right]$ for NTS.

\begin{tabular}{|c|c|}
\hline $\mathrm{S}(1)-\mathrm{N}(1)$ & $1.6860(14)$ \\
\hline $\mathrm{S}(1)-\mathrm{C}(5)$ & $1.696(2)$ \\
\hline $\mathrm{O}(1)-\mathrm{C}(1)$ & $1.197(2)$ \\
\hline $\mathrm{O}(2)-\mathrm{C}(4)$ & $1.198(2)$ \\
\hline $\mathrm{N}(1)-\mathrm{C}(4)$ & $1.400(2)$ \\
\hline $\mathrm{N}(1)-\mathrm{C}(1)$ & $1.404(2)$ \\
\hline $\mathrm{N}(2)-\mathrm{C}(5)$ & $1.130(3)$ \\
\hline $\mathrm{C}(1)-\mathrm{C}(2)$ & $1.499(3)$ \\
\hline$C(2)-C(3)$ & $1.516(3)$ \\
\hline $\mathrm{C}(2)-\mathrm{H}(2 \mathrm{~A})$ & 0.9700 \\
\hline $\mathrm{C}(2)-\mathrm{H}(2 \mathrm{~B})$ & 0.9700 \\
\hline$C(3)-C(4)$ & $1.499(2)$ \\
\hline $\mathrm{C}(3)-\mathrm{H}(3 \mathrm{~A})$ & 0.9700 \\
\hline $\mathrm{C}(3)-\mathrm{H}(3 \mathrm{~B})$ & 0.9700 \\
\hline $\mathrm{N}(1)-\mathrm{S}(1)-\mathrm{C}(5)$ & $98.93(8)$ \\
\hline $\mathrm{C}(4)-\mathrm{N}(1)-\mathrm{C}(1)$ & $113.42(14)$ \\
\hline $\mathrm{C}(4)-\mathrm{N}(1)-\mathrm{S}(1)$ & $121.40(12)$ \\
\hline $\mathrm{C}(1)-\mathrm{N}(1)-\mathrm{S}(1)$ & $124.96(13)$ \\
\hline $\mathrm{O}(1)-\mathrm{C}(1)-\mathrm{N}(1)$ & $123.95(18)$ \\
\hline $\mathrm{O}(1)-\mathrm{C}(1)-\mathrm{C}(2)$ & $129.03(17)$ \\
\hline $\mathrm{N}(1)-\mathrm{C}(1)-\mathrm{C}(2)$ & $107.01(15)$ \\
\hline $\mathrm{C}(1)-\mathrm{C}(2)-\mathrm{C}(3)$ & $106.32(14)$ \\
\hline $\mathrm{C}(1)-\mathrm{C}(2)-\mathrm{H}(2 \mathrm{~A})$ & 110.5 \\
\hline $\mathrm{C}(3)-\mathrm{C}(2)-\mathrm{H}(2 \mathrm{~A})$ & 110.5 \\
\hline $\mathrm{C}(1)-\mathrm{C}(2)-\mathrm{H}(2 \mathrm{~B})$ & 110.5 \\
\hline $\mathrm{C}(3)-\mathrm{C}(2)-\mathrm{H}(2 \mathrm{~B})$ & 110.5 \\
\hline $\mathrm{H}(2 \mathrm{~A})-\mathrm{C}(2)-\mathrm{H}(2 \mathrm{~B})$ & 108.7 \\
\hline$C(4)-C(3)-C(2)$ & $105.72(15)$ \\
\hline $\mathrm{C}(4)-\mathrm{C}(3)-\mathrm{H}(3 \mathrm{~A})$ & 110.6 \\
\hline $\mathrm{C}(2)-\mathrm{C}(3)-\mathrm{H}(3 \mathrm{~A})$ & 110.6 \\
\hline $\mathrm{C}(4)-\mathrm{C}(3)-\mathrm{H}(3 \mathrm{~B})$ & 110.6 \\
\hline $\mathrm{C}(2)-\mathrm{C}(3)-\mathrm{H}(3 \mathrm{~B})$ & 110.6 \\
\hline $\mathrm{H}(3 \mathrm{~A})-\mathrm{C}(3)-\mathrm{H}(3 \mathrm{~B})$ & 108.7 \\
\hline
\end{tabular}




$\begin{array}{ll}\mathrm{O}(2)-\mathrm{C}(4)-\mathrm{N}(1) & 123.56(17) \\ \mathrm{O}(2)-\mathrm{C}(4)-\mathrm{C}(3) & 129.02(19) \\ \mathrm{N}(1)-\mathrm{C}(4)-\mathrm{C}(3) & 107.42(15) \\ \mathrm{N}(2)-\mathrm{C}(5)-\mathrm{S}(1) & 177.9(2)\end{array}$

Symmetry transformations used to generate equivalent atoms: 
Table S7. Anisotropic displacement parameters $\left(\AA^{2} \times 10^{3}\right)$ for NTS. The anisotropic displacement factor exponent takes the form: $-2 \pi^{2}\left[h^{2} a^{* 2} U^{11}+\ldots+2 h k a^{*} b^{*} U^{12}\right]$

\begin{tabular}{lcccccc}
\hline & $\mathrm{U}^{11}$ & $\mathrm{U}^{22}$ & $\mathrm{U}^{33}$ & $\mathrm{U}^{23}$ & $\mathrm{U}^{13}$ & $\mathrm{U}^{12}$ \\
\hline $\mathrm{S}(1)$ & $43(1)$ & $63(1)$ & $40(1)$ & $6(1)$ & $-6(1)$ & $-16(1)$ \\
$\mathrm{O}(1)$ & $74(1)$ & $55(1)$ & $49(1)$ & $-12(1)$ & $-3(1)$ & $13(1)$ \\
$\mathrm{O}(2)$ & $49(1)$ & $61(1)$ & $89(1)$ & $-18(1)$ & $11(1)$ & $10(1)$ \\
$\mathrm{N}(1)$ & $33(1)$ & $40(1)$ & $36(1)$ & $5(1)$ & $1(1)$ & $-3(1)$ \\
$\mathrm{N}(2)$ & $59(1)$ & $84(1)$ & $48(1)$ & $16(1)$ & $1(1)$ & $-9(1)$ \\
$\mathrm{C}(1)$ & $44(1)$ & $44(1)$ & $30(1)$ & $5(1)$ & $-1(1)$ & $9(1)$ \\
$\mathrm{C}(2)$ & $35(1)$ & $56(1)$ & $50(1)$ & $14(1)$ & $7(1)$ & $5(1)$ \\
$\mathrm{C}(3)$ & $39(1)$ & $46(1)$ & $59(1)$ & $4(1)$ & $-3(1)$ & $-6(1)$ \\
$\mathrm{C}(4)$ & $36(1)$ & $41(1)$ & $46(1)$ & $1(1)$ & $-2(1)$ & $2(1)$ \\
$\mathrm{C}(5)$ & $39(1)$ & $48(1)$ & $43(1)$ & $2(1)$ & $2(1)$ & $-9(1)$ \\
& & & & & & \\
\hline
\end{tabular}


Table S8. Hydrogen coordinates $\left(\mathrm{x} 10^{4}\right)$ and isotropic displacement parameters $\left(\AA^{2} \mathrm{x}\right.$ $10^{3}$ ) for NTS.

\begin{tabular}{lrrrr}
\hline & $x$ & $y$ & $z$ & U(eq) \\
\hline $\mathrm{H}(2 \mathrm{~A})$ & 11672 & 3731 & 2749 & 57 \\
$\mathrm{H}(2 \mathrm{~B})$ & 12105 & 2748 & 3533 & 57 \\
$\mathrm{H}(3 \mathrm{~A})$ & 11369 & 5332 & 4191 & 57 \\
$\mathrm{H}(3 \mathrm{~B})$ & 10959 & 6341 & 3405 & 57 \\
\hline
\end{tabular}


Table S9. Torsion angles $\left[{ }^{\circ}\right]$ for NTS.

\begin{tabular}{lc}
\hline $\mathrm{C}(5)-\mathrm{S}(1)-\mathrm{N}(1)-\mathrm{C}(4)$ & $-81.76(15)$ \\
$\mathrm{C}(5)-\mathrm{S}(1)-\mathrm{N}(1)-\mathrm{C}(1)$ & $103.91(14)$ \\
$\mathrm{C}(4)-\mathrm{N}(1)-\mathrm{C}(1)-\mathrm{O}(1)$ & $-176.13(16)$ \\
$\mathrm{S}(1)-\mathrm{N}(1)-\mathrm{C}(1)-\mathrm{O}(1)$ & $-1.4(2)$ \\
$\mathrm{C}(4)-\mathrm{N}(1)-\mathrm{C}(1)-\mathrm{C}(2)$ & $2.67(18)$ \\
$\mathrm{S}(1)-\mathrm{N}(1)-\mathrm{C}(1)-\mathrm{C}(2)$ & $177.39(11)$ \\
$\mathrm{O}(1)-\mathrm{C}(1)-\mathrm{C}(2)-\mathrm{C}(3)$ & $177.95(18)$ \\
$\mathrm{N}(1)-\mathrm{C}(1)-\mathrm{C}(2)-\mathrm{C}(3)$ & $-0.77(18)$ \\
$\mathrm{C}(1)-\mathrm{C}(2)-\mathrm{C}(3)-\mathrm{C}(4)$ & $-1.2(2)$ \\
$\mathrm{C}(1)-\mathrm{N}(1)-\mathrm{C}(4)-\mathrm{O}(2)$ & $177.35(18)$ \\
$\mathrm{S}(1)-\mathrm{N}(1)-\mathrm{C}(4)-\mathrm{O}(2)$ & $2.4(3)$ \\
$\mathrm{C}(1)-\mathrm{N}(1)-\mathrm{C}(4)-\mathrm{C}(3)$ & $-3.4(2)$ \\
$\mathrm{S}(1)-\mathrm{N}(1)-\mathrm{C}(4)-\mathrm{C}(3)$ & $-178.38(12)$ \\
$\mathrm{C}(2)-\mathrm{C}(3)-\mathrm{C}(4)-\mathrm{O}(2)$ & $-178.1(2)$ \\
$\mathrm{C}(2)-\mathrm{C}(3)-\mathrm{C}(4)-\mathrm{N}(1)$ & $2.7(2)$ \\
& \\
\hline
\end{tabular}

Symmetry transformations used to generate equivalent atoms: 


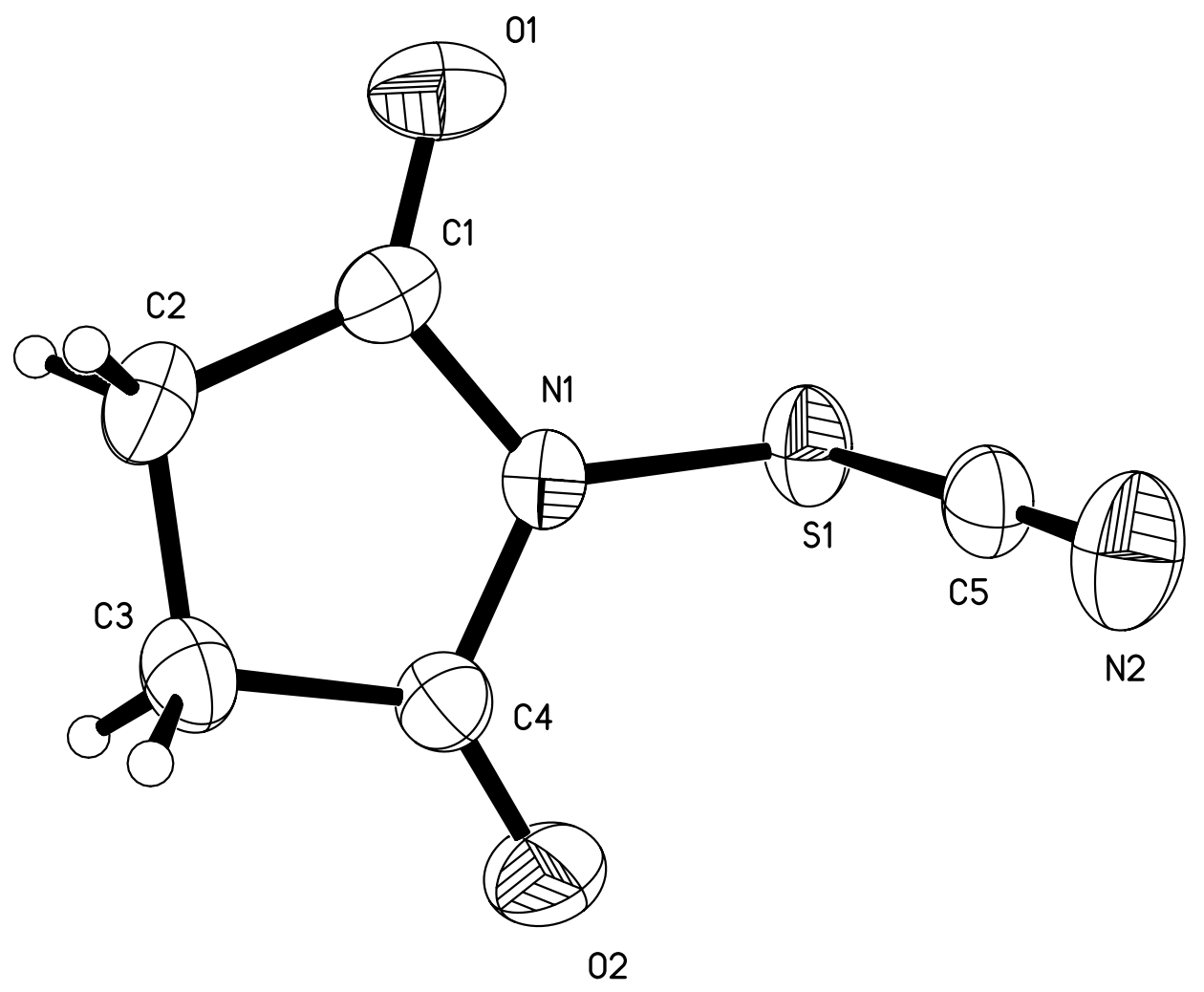

Figure S1. Thermal ellipoid drawing of the crystal structure of NTS at the $50 \%$ level with the labeling scheme. 


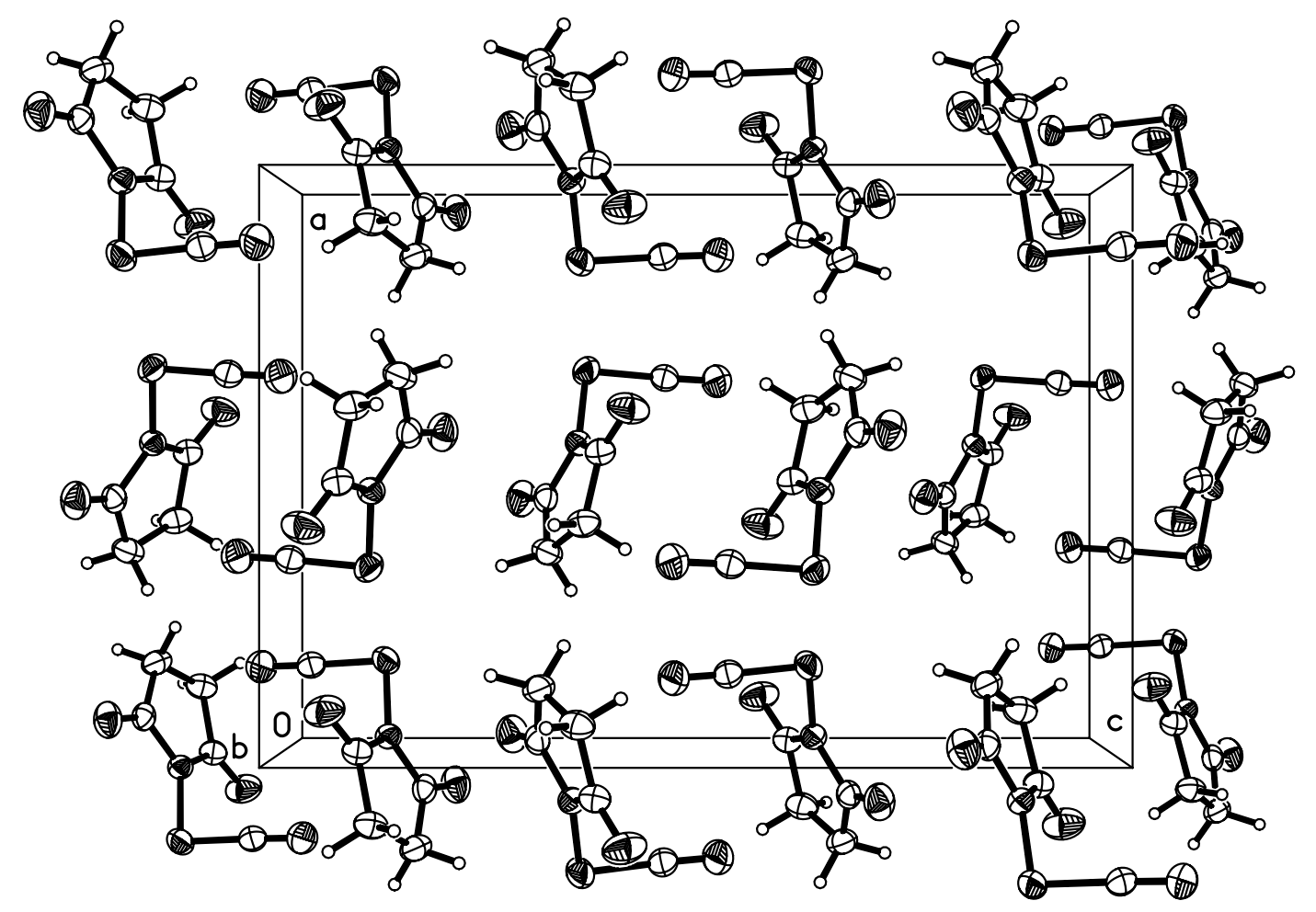

Figure S2. Crystal packing diagram of the crystal structure of NTS as viewed along the b axis. 